\title{
Evaluación numérica del efecto del metal de soldadura sobre el factor de constricción en probetas $\mathrm{C}(\mathrm{T})$ bimetálicas ${ }^{(\bullet)}$
}

\author{
J.R. Donoso*, A. Ortiz** y F. Labbé**
}

\begin{abstract}
Resumen El efecto del metal de soldadura sobre la constricción fuera-del-plano en probetas $\mathrm{C}(\mathrm{T})$ bimetálicas fue estudiado utilizando la Ecuación del Formato Común (EFC) y su factor de constricción asociado, $\Omega^{*}$, en función del cociente espesor de probeta a ligamento, $\mathrm{B} / \mathrm{b}$. Se compararon los valores de $\Omega^{*}$ para probetas con sólo un material (metal base), y para probetas bimetálicas (metal base más metal de soldadura), constatándose que la presencia de metal de soldadura hace disminuir el grado de constricción en las probetas bimetálicas, con respecto a las probetas con sólo metal base. Este efecto se ve atenuado para grieta profunda, dando resultados similares para $\Omega^{*}$ en las probetas con sólo metal base y bimetálica.
\end{abstract}

Palabras clave Fractura elasto-plástica. Formato común. Factor de constricción. Tenacidad a la fractura.

\section{Numerical evaluation of the effect of the weld metal on the constraint factor in bi-metal $C(T)$ specimens}

\begin{abstract}
The effect of the weld metal on the out-of-plane constraint in $\mathrm{C}(\mathrm{T})$ bi-material specimens was studied using the Common Format Equation (CFE) and its associated constraint factor, $\Omega^{*}$, as a function of the thickness-to-ligament ratio, B/b. The values of $\Omega^{*}$ for onematerial specimens (base metal) and bi-material specimens (base metal plus weld metal) were compared. The presence of weld metal lowers the degree of constraint in the bimaterial specimens in relation to the base metal specimens, for a given $\mathrm{B} / \mathrm{b}$ value. This effect is attenuated for deeply cracked specimens, the $\Omega *$ values being similar for the onematerial and the bi-material specimens.
\end{abstract}

Keywords

Elastic-plastic fracture. Common format. Constraint factor. Fracture toughness.

\section{INTRODUCCIÓN}

El efecto de la constricción sobre la tenacidad a la fractura de materiales dúctiles ha sido extensamente estudiado en las últimas dos décadas. A modo de ejemplo, la curva J-R, tomada como una medida de la tenacidad a la fractura, depende, fuertemente, de las dimensiones en el plano de la probeta, del modo de carga y de la longitud de grieta. Así, una probeta con grieta profunda dará lugar a una tenacidad aparente que es menor que la mostrada por una probeta con grieta superficial. De igual manera, una probeta con grieta centrada en tensión, $M(T)$, generará una curva J-R más alta que una probeta de flexión, $\mathrm{SE}(\mathrm{B})^{[1]}$.
No sólo la tenacidad a la fractura de un material es afectada por el grado de constricción. Las funciones de calibración, relaciones carga-desplazamiento, también se ven afectadas por la constricción impuesta sobre la probeta. En este sentido, la constricción influye sobre las propiedades de fractura y sobre las características de deformación de maneras opuestas. Un elevado grado de constricción logra bajar la curva J-R, pero eleva la curva carga-desplazamiento de la probeta. A la inversa, un bajo grado de constricción hace elevar la curva $\mathrm{J}-\mathrm{R}$ y bajar la curva carga-desplazamiento de la probeta. Desde el punto de vista de un enfoque 2-D, el máximo grado de constricción está asociado con un estado de deformación plana, en tanto

(•) Trabajo recibido el día 10 de marzo de 2003 y aceptado en su forma final el día 16 de septiembre de 2003.

(*) Departamento Ciencia de Materiales. Universidad Técnica Federico Santa María. Av. España 1680. Valparaíso, CHILE

(*) Departamento de Mecánica. Universidad Técnica Federico Santa María. Av. España 1680. Valparaíso, CHILE 
el mínimo está asociado con un estado de tensión plana.

En probetas de fractura fabricadas con materiales homogéneos (probetas con sólo un material), los efectos de constricción se deben principalmente a las dimensiones (longitud de grieta y/o espesor de probeta) y a la geometría de la probeta. La influencia se debe, entonces, a constricción por tamaño y por geometría, respectivamente, siendo muchas veces difícil separar los efectos. Ejemplos de este tipo de comportamiento abundan en la literatura $^{[2}$ y 3$]$.

Para probetas bimetálicas, en cambio, como las probetas fabricadas a partir de una unión soldada, el efecto es una combinación de la constricción por tamaño, por geometría, y por material. En este caso, los efectos de tamaño y/o geometría se ven modificados por la presencia de la grieta en una porción de material, el metal de soldadura, MS, cuyas propiedades en deformación son diferentes a las del metal base, MB. No debe sorprender, entonces, que el parámetrò fundamental que se invoca cuando se plantean las soluciones para probetas con metal de soldadura, es el desajuste del límite elástico. Este parámetro está definido como el cociente del límite elástico de MS al límite elástico de MB. Designando al desajuste como MM, éste queda definido por la siguiente relación:

$$
\mathrm{MM}=\frac{\sigma_{0}^{\mathrm{MS}}}{\sigma_{0}^{\mathrm{MS}}}
$$

donde, $\sigma_{0}^{\mathrm{MS}}$ y $\sigma_{0}^{\mathrm{MS}}$ son los límites elásticos de MS y $\mathrm{MB}$, respectivamente. La unión soldada estará sobre-ajustada si $\sigma_{0}^{\mathrm{MS}}>\sigma_{0}^{\mathrm{MS}}$; y sub-ajustada, si $\sigma_{0}^{\mathrm{MS}}<$ $\sigma_{0}^{\mathrm{MS}}$. La tercera posibilidad, es decir $\sigma_{0}^{\mathrm{MS}}=\sigma_{0}^{\mathrm{MS}}$, será designada, simplemente, como ajustada.

El efecto del material sobre la constricción en uniones soldadas, se ha analizado tanto del punto de vista experimental como numérico ${ }^{[4-6]}$. La conclusión principal es que, la presencia de un material en el lugar donde está la grieta (MS), con propiedades diferentes a las del material que lo rodea (MB), altera el grado de constricción. Considerando que, ésta, es afectada por la geometría y las dimensiones en el plano, la constricción por el material es, eventualmente, separable de otras formas de constricción ${ }^{[7]}$.

Dos enfoques diferentes se han utilizado, hasta ahora, para analizar el efecto que la presencia de metal de soldadura tiene sobre la constricción. En el primero de ellos, Kim et al..$^{[8 \text { y } 9]}$ usan el Engineering Treatment Model (ETM) y obtienen soluciones para la carga correspondiente al límite elástico en función de la geometría, grado de desajuste, ubicación de la grieta (MS o interfaz MB/MS), ancho del cordón de soldadura y tamaño de grieta. Sus cálculos por elementos finitos emplean modelos 2 $\mathrm{D}$, se analizan tanto en deformación plana como en tensión plana y están basados en plasticidad sin endurecimiento ${ }^{[9]}$.

Un segundo enfoque es el, así llamado, J-Q-M, propuesto recientemente por Thaulow et al. ${ }^{[7]}$, que constituye una extensión a sistemas bimateriales y trimateriales del enfoque $\mathrm{J}-\mathrm{Q}$, originalmente desarrollado por O'Dowd y Shih para materiales homogéneos ${ }^{[3]}$. Con esta perspectiva, $Q$ cuantifica la constricción debido a la geometría y $\mathrm{M}$ la constricción debido al desajuste de material, efectos que pueden ser tratados en forma independiente en algunos casos. En otros casos, $\mathrm{M}$ presenta una cierta dependencia de la geometría atribuible al modo de carga, relación de desajuste y posición de la grieta ${ }^{[10]}$.

El presente trabajo analiza los efectos que una unión soldada sobre-ajustada tiene sobre la constricción en probetas de fractura $\mathrm{C}(\mathrm{T})$, utilizando una opción diferente: la Ecuación del Formato Común (EFC), de Donoso y Landes ${ }^{[11]}$ y su factor de constricción asociado, $\Omega^{*}$. Los fundamentos de la EFC y el factor de constricción $\Omega^{*}$ serán presentados a continuación.

\section{EL FACTOR DE CONSTRICCIÓN DEL FORMATO COMÚN, $\Omega^{*}$}

En una metodología de análisis de fractura dúctil, se requiere tanto la tenacidad a la fractura como las funciones de calibración, esto es, las relaciones P-v, propias del material. Históricamente, el énfasis ha estado puesto, más en evaluar la tenacidad a la fractura que en determinar las relaciones carga, vs. desplazamiento.

Donoso y Landes desarrollaron la Ecuación del Formato Común, EFC ${ }^{[11 \text { y } 12]}$, y el Formato Conciso, $\mathrm{FC}^{[13]}$, para permitir una descripción completa, al menos para deformaciones pequeñas, de las relaciones $\mathrm{P}-\mathrm{v}$ para componentes agrietados. El FC permite describir las funciones de calibración en la región elástica, en tanto la EFC provee las relaciones carga-desplazamiento en la región de comportamiento no-lineal. El enfoque de la EFC y su factor de constricción asociado, $\Omega^{*}$, se presentó previamente ${ }^{[14]}$, por lo que no será discutido aquí. 
Sólo señalaremos que, en el enfoque de la EFC ${ }^{[11]}$, la carga, $\mathrm{P}$, se expresa como el producto de tres términos, como lo muestra la ecuación (2):

$$
P=\Omega * G\left(\frac{b}{W}\right) \cdot H\left(\frac{v_{p l}}{W}\right)
$$

donde, $G$ es una función propia de la geometría que depende del tamaño del ligamento, $\mathrm{H}$ es una función de endurecimiento dependiente de la componente plástica del desplazamiento y $\Omega *$ es el factor de constricción de la EFC.

Las funciones $\mathrm{G}$ y $\mathrm{H}$ son bien conocidas ${ }^{[12]}$ y ya se han sido planteado en un trabajo anterior ${ }^{[14]}$. La evaluación y/o predicción de $\Omega *$, en cambio, siempre ha sido una tarea difícil. Es un hecho reconocido que el grado de constricción influye, tanto sobre la tenacidad a la fractura como sobre las relaciones carga-desplazamiento. No obstante, en cuanto a la relevancia del factor de restricción, sólo recientemente, se ha analizado cómo depende $\Omega^{*}$ de las dimensiones de la probeta de fractura ${ }^{[14]}$ y cuán útil es $\Omega^{*}$ para realizar estimaciones de $\mathrm{J}$.

El enfoque bi-dimensional al problema de la constricción en el plano, da dos valores para el factor $\Omega^{*}$. Así, probetas planas de fractura tienen un comportamiento 2-D que es, o deformación plana o tensión plana. En consecuencia, el factor de constricción de la Ec. (2) adopta un valor específico: en el enfoque de la EFC, $\Omega^{*}$ es igual a 0,3638 para deformación plana e igual a 0,2678 para tensión plana ${ }^{[2}$ y 11]. Valores experimentales y numéricos entre estos dos extremos son factibles $\mathrm{e}$ indicarían una tendencia para el comportamiento de la probeta, el cual depende del tipo de probeta (geometría), condiciones de carga (tensión vs. doblado), dimensiones en el plano (longitud de grieta y ancho), y espesor de probeta ${ }^{[14]}$.

Donoso y Landes propusieron un método para poder evaluar experimentalmente el grado de constricción de una probeta plana de fractura ${ }^{[2]}$. El método consiste en examinar la relación carga normalizada, vs. desplazamiento plástico normalizado, basada en una ley potencial para $\mathrm{H}^{[14]}$, con lo cual:

$$
\mathrm{P}_{\mathrm{N}}=\frac{\mathrm{P}}{\mathrm{G}}=\Omega * \sigma * \mathrm{v}_{\mathrm{N}}^{1 / \mathrm{n}}=\mathrm{D} \mathrm{v}_{\mathrm{N}}^{1 / \mathrm{n}}
$$

Un análisis completo de la información carga, vs., desplazamiento, experimental o numérica, en el formato $\mathrm{P}_{\mathrm{N}}$, vs. $\mathrm{v}_{\mathrm{N}}$, dará el valor de $\mathrm{D}$, que es, a su vez, igual a $\Omega * \sigma^{*}$. Luego el valor del factor de constricción de la EFC puede ser calculado a través del conocimiento de $\sigma^{*}$ y $D$, como lo muestra la ecuación (4):

$$
\Omega^{*}=\mathrm{D} / \sigma^{*}
$$

En el trabajo inicial de Donoso y Landes se construyó un mapa de comportamiento deformación plana, vs. tensión plana, en función del cociente espesor a ligamento, $\mathrm{B} / \mathrm{b}$, para probetas $\mathrm{C}(\mathrm{T})$ de diferentes espesores y dimensiones en el plano ${ }^{[2]}$. En el trabajo de la referencia ${ }^{[2]}$, los valores de $\Omega *$ fueron calculados con la ecuación (4). Una forma alternativa de evaluar $\Omega^{*}$ es a través de la carga límite, $\mathrm{P}_{\mathrm{o}}$. En efecto, la carga para un desplazamiento normalizado $\mathrm{v}_{\mathrm{N}}=0,002^{[12]}$, resulta ser igual a

$$
\mathrm{P}_{\mathrm{o}}=\Omega * \mathrm{G} \sigma_{\mathrm{o}}
$$

Despejando el factor de constricción, $\Omega^{*}$, de la ecuación (5), se obtiene

$$
\Omega^{*}=\mathrm{P}_{\mathrm{o}} / \mathrm{G} \sigma_{\mathrm{o}}=\mathrm{P}_{\mathrm{N}, \mathrm{o}} / \sigma_{\mathrm{o}}
$$

Trabajos posteriores han demostrado que el factor $\Omega^{*}$ puede variar con la geometría, con las dimensiones en el plano y con la deformación plásti$\mathrm{ca}^{[14}$ y 15] . Con el objeto de obtener $\Omega^{*}$ para las probetas con sólo $\mathrm{MB}$, y para las que tienen MS, a partir de resultados numéricos, se usará en este trabajo el enfoque basado en la evaluación de la carga límite, dado por la ecuación (6), es decir,

$$
\Omega_{\mathrm{i}}^{*}=\left(\mathrm{P}_{\mathrm{N}, \mathrm{o}} / \sigma_{\mathrm{o}}\right)_{\mathrm{i}}
$$

donde, i representa MB o MS. Este enfoque alternativo procura eliminar los efectos que, de otra manera, puede tener la deformación plástica sobre los valores del coeficiente $\mathrm{D}^{[15]}$.

En este artículo se mostrará cómo la presencia de un volumen de material, MS, donde se ubica la grieta, con propiedades mecánicas diferentes a las del material que lo rodea, $\mathrm{MB}$, metal base, afecta al factor de constricción, tal como éste está definido por el parámetro $\Omega^{*}$ de la EFC. Este efecto se ha denominado en este trabajo como la constricción por el material, y será presentado en función del cociente $\mathrm{B} / \mathrm{b}$, el espesor equivalente ${ }^{[14]}$. Los modelos y la estrategia del análisis por elementos finitos se explicarán a continuación. 


\section{LOS MODELOS DE ELEMENTOS FINITOS}

Se simuló el comportamiento no-lineal de probetas de fractura del tipo $C(T)$, con grieta estacionaria, sólo de metal base (MB) y bimetálicas (designadas, en adelante, como MS), usando el programa de elementos finitos ADINA ${ }^{[16]}$. La figura 1 muestra un esquema de la probeta $\mathrm{C}(\mathrm{T})$ con un cordón de soldadura en su centro, que es el lugar donde se ubica la grieta; la probeta $\mathrm{MB}$ es aquella en la cual no hay MS, es decir, $2 \mathrm{~h}=0$.

Para simular el cordón de soldadura en la probeta MS, la malla se construyó de dos zonas: una, donde está ubicada la grieta, tiene las propiedades mecánicas de MS, en tanto el resto de la probeta tiene propiedades de MB. En este modelo no se consideraron efectos de dilución (las propiedades mecánicas cambian abruptamente en la interfaz recta MB-MS), ni la existencia de la ZAT (zona afectada térmicamente). Debido a la simetría de la probeta, se utilizó sólo un cuarto de ella, cuyo mallado se muestra, en perspectiva, en la figura 2. Por razones de simplicidad, no se representaron los agujeros (ignorar los agujeros afecta los resultados sólo para probetas con tamaño de grieta pequeño, v.g., a/W $\leq 0,25^{[4]}$ ), y el corte para generar la grieta se modeló como una ranura simple, con un radio de curvatura en la punta, de $0,040 \mathrm{~mm}$.

Los modelos construidos tienen, en promedio, 6.425 elementos sólidos 3-D de 8 nodos, con un total de alrededor de 7.700 nodos. Los elementos ubicados cerca de la punta de la grieta disminuyen en tamaño en la medida que se acercan a aquélla. La punta tiene un radio de curvatura de $0,040 \mathrm{~mm}$,

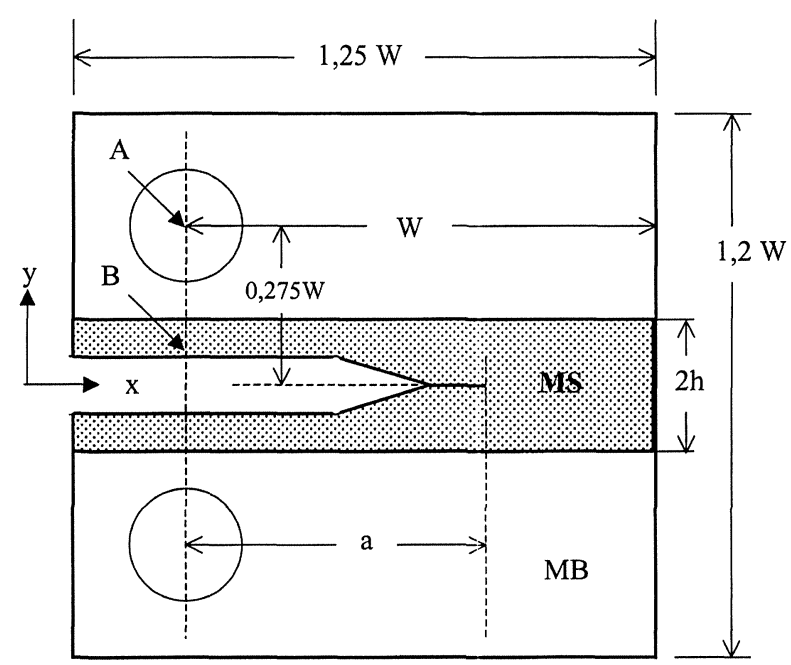

Figura 1. Esquema de la probeta $C(T)$ con un cordón de soldadura (MS).

Figure 1. Schematic of the $C(T)$ specimen with a weld (MS).

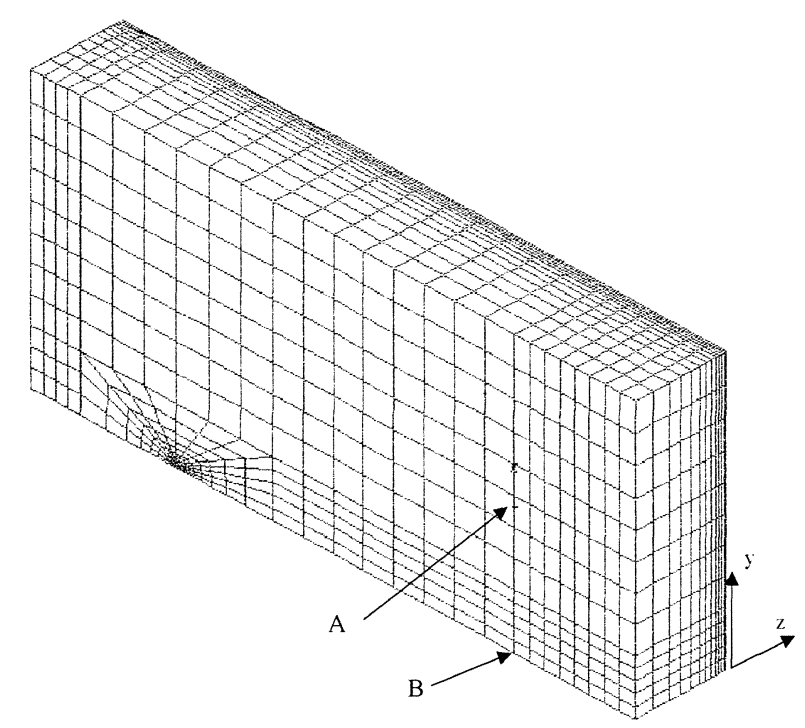

Figura 2. Malla típica para la probeta $C(T)$.

Figure 2. Typical mesh for the $C(T)$ specimen.

y los elementos más cercanos tienen dimensiones de $0,005 \times 0,005 \mathrm{~mm}^{2}$. La figura 3 muestra una vista ampliada de la malla empleada en la cercanía de la punta de la grieta, donde se ubicaron alrededor de 3/4 del total de los elementos.

Para los modelos se usó un valor del límite elástico de $240 \mathrm{MPa}$ para MB y de $300 \mathrm{MPa}$ para MS, dando un sobre-ajuste de valor $\mathrm{MM}=1,25$. El módulo de Young y el coeficiente de Poisson, comunes a ambos materiales, tienen los valores 210 $\mathrm{GPa}$ y 0,30 , respectivamente. El análisis numérico consideró el criterio de von Mises, no-linealidad del material y formulación lagrangiana actualizada, para incluir adecuadamente el efecto de grandes deformaciones. Las simulaciones se ejecutaron bajo la condición de desplazamientos prescritos, utilizando entre 80 y 100 pasos para un desplazamien-

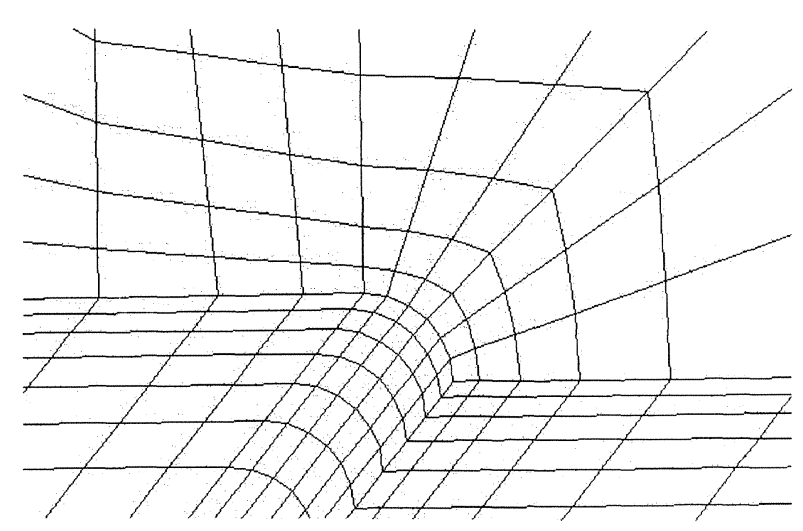

Figura 3. Detalle de la malla en la punta de la grieta.

Figure 3. Detail of the mesh at the crack tip. 
to total del punto de aplicación de carga (punto A en la Fig. 1) de $4 \mathrm{~mm}$.

En la figura 1, todas las dimensiones en el plano son proporcionales a la probeta estándar según ASTM E1820 ${ }^{[17]}$, con $\mathrm{W}=50 \mathrm{~mm}$, independientes del espesor utilizado. En esta probeta, $2 \mathrm{~h}$ denota la porción de metal de soldadura, definiendo la fracción de volumen de MS como $\mathrm{r}=2 \mathrm{~h} /(1,2 \mathrm{~W})$. La probeta con MS tiene $r=0,20$, es decir, $h=6$ $\mathrm{mm}$, en tanto aquella con sólo $\mathrm{MB}$ tiene $\mathrm{r}=0$. Dada la simetría de la probeta $\mathrm{C}(\mathrm{T})$, se usó sólo un cuarto de ella. Se hace hincapié que la grieta está en MS y, por lo tanto, no es una grieta interfacial.

Los modelos construidos tienen longitudes normalizadas de grieta, a/W, con valores $0,4,0,5,0,6$, 0,7 y 0,8 , correspondiendo las tres últimas a lo que se denomina "grieta profunda". Los valores utilizados del espesor $B$, manteniendo las dimensiones en el plano constantes, son 3,$125 ; 6,25 ; 12,5 ; 18,75$ y $25 \mathrm{~mm}$, dando así lugar a los valores del cociente $\mathrm{B} / \mathrm{b}$ listados en la tabla I. Los 25 valores tabulados sólo dan 17 valores diferentes, puesto que en algunos casos, un valor dado de $\mathrm{B} / \mathrm{b}$ se puede obtener por dos o más combinaciones de espesor y tamaño de grieta.

\section{RESULTADOS Y DISCUSIÓN}

Los resultados se extrajeron en la forma de carga vs. desplazamiento total de un punto sobre la línea de carga. En la probeta C(T) (Fig. 1), el desplazamiento del punto de aplicación de carga en un ensayo real se mide en $A$, en tanto que en un punto equivalente a $\mathrm{B}$ se mide la cantidad denominada cmod [17]. Los desplazamientos verticales de los puntos A y B son idénticos para tamaños de grieta $\mathrm{a} / \mathrm{W}$, y de cordón, $2 \mathrm{~h}$, como los usados en este trabajo. Las diferencias en desplazamiento entre ambos puntos son notorias, en cambio, para tamaños

Tabla I. Valores de B/b

Table I. Values of $B / b$

\begin{tabular}{lccccc}
\hline $\mathrm{a} / \mathrm{W}$ & \multicolumn{5}{c}{$\mathrm{B}, \mathrm{mm}$} \\
\cline { 2 - 6 } & 3,125 & 6,25 & 12,5 & 18,75 & 25 \\
\hline 0,4 & 0,104 & 0,208 & 0,417 & 0,625 & 0,833 \\
0,5 & 0,125 & 0,250 & 0,500 & 0,750 & 1,000 \\
0,6 & 0,156 & 0,313 & 0,625 & 0,938 & 1,250 \\
0,7 & 0,208 & 0,417 & 0,833 & 1,250 & 1,667 \\
0,8 & 0,313 & 0,625 & 1,250 & 1,875 & 2,500 \\
\hline
\end{tabular}

Rev. Metal. Madrid 39 (2003) 357-366 de grieta pequeños, es decir, a/W $<0,25$, y/o tamaños de cordón $2 h<3 \mathrm{~mm}$.

La componente plástica del desplazamiento, variable necesaria para la aplicación de la EFC, se obtuvo restando al desplazamiento total la componente elástica, obtenida por medio de la flexibilidad correspondiente a cada espesor y longitud de grieta. En adelante, se entenderá que desplazamiento significa componente plástica del desplazamiento.

Las figuras 4, 5 y 6 muestran ejemplos de los efectos de la constricción por el tamaño de grieta,

$\mathrm{P} / \mathrm{B}, \mathrm{N} / \mathrm{mm}$

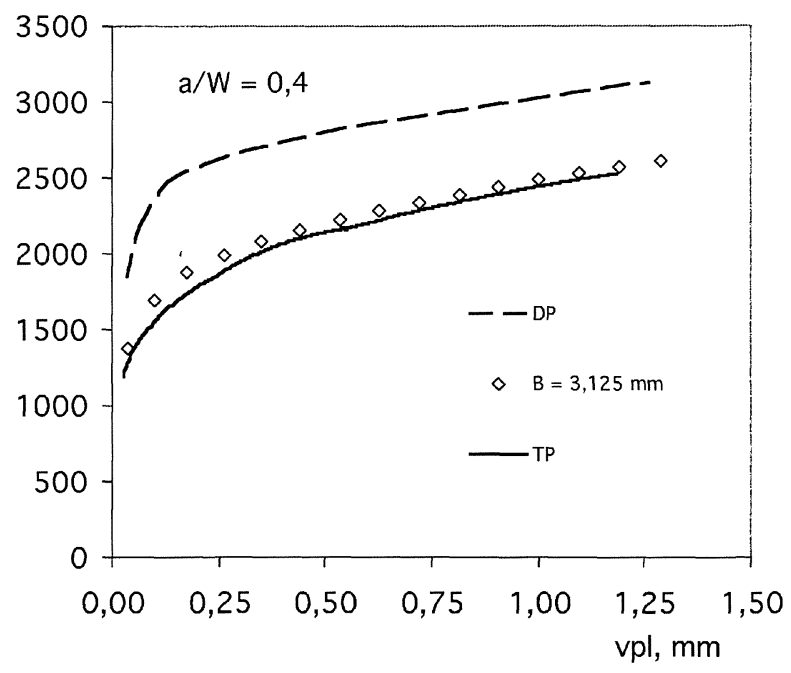

Figura 4. Curvas $P / B$, vs. vpl para $a / W=0,4$ y $B=3,125$ $\mathrm{mm}$.

Figure 4. $P / B$, vs. $v_{p} /$ curves for $a / W=0,4$ and $B=3,125 \mathrm{~mm}$.

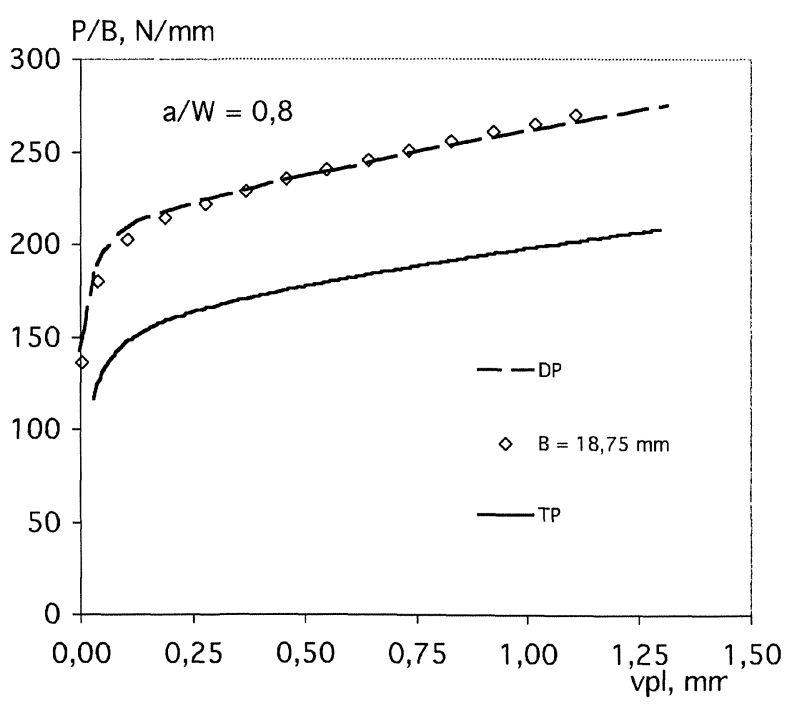

Figura 5. Curvas $P / B$, vs. $v_{p}$ para $a / W=0,8$ y $B=18,75$ $\mathrm{mm}$.

Figure 5. $P / B$, vs. $v p /$ curves for $a / W=0,8$ and $B=18,75 \mathrm{~mm}$. 
$\mathrm{P} / \mathrm{B}, \mathrm{N} / \mathrm{mm}$

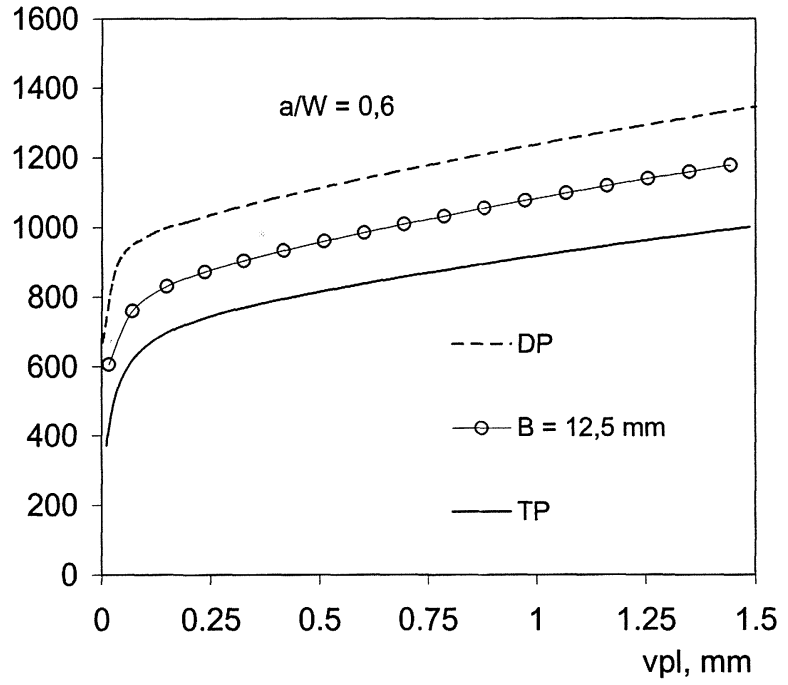

Figura 6. Curvas $P / B$, vs. vpl para $a / W=0,6$ y $B=12,5$ $\mathrm{mm}$.

Figure 6. $P / B$, vs. vpl curves for $a / W=0,6$ and $B=12,5 \mathrm{~mm}$.

constricción en el plano y por el espesor, constricción fuera del plano, para probetas con sólo MB. En la figura 4 se muestra las curvas carga por unidad de espesor, $\mathrm{P} / \mathrm{B}$, vs. el desplazamiento, para $\mathrm{a} / \mathrm{W}=0,4$. La curva superior corresponde a la situación en deformación plana (DP), en tanto la inferior, corresponde a tensión plana (TP). La curva propia de la probeta $3-\mathrm{D}$, con espesor 3,125 $\mathrm{mm}$, está próxima a la curva TP. Por ello, su grado de constricción es cercano al de tensión plana. El valor de espesor equivalente de esta probeta, $\mathrm{B} / \mathrm{b}$, es 0,104 .

La figura 5 , en tanto, muestra una situación similar de comparación, pero para a/W $=0,8$ y mayor espesor. En este caso, la curva de la probeta 3 - $\mathrm{D}$, con $\mathrm{B}=18,75 \mathrm{~mm}$, es prácticamente coincidente con la curva DP, siendo su grado de constricción cercano al de deformación plana. Para esta probeta, el valor de espesor equivalente es 1,875. Las curvas de las figuras 4 y 5 son ejemplos típicos del efecto de la constricción por tamaño, incluyendo longitud de grieta y espesor.

La figura 6 muestra lo que ocurre en una probeta con un tamaño de grieta y espesor de valores intermedios; en este caso, a/ $\mathrm{W}=0,6, \mathrm{y} B=12,5 \mathrm{~mm}$, con $\mathrm{B} / \mathrm{b}=0,625$. La probeta con valores intermedios de a/W y B se ubica entre las curvas correspondientes a tensión plana y deformación plana. Entonces, en una situación como la mostrada en la figura 6 , uno debe preguntarse ¿cuál es el grado de constricción de esta probeta? De manera cualitativa, la respuesta es: ni tensión plana ni deforma- ción plana. De forma cuantitativa, la respuesta la dará el factor de constricción de la EFC, $\Omega^{*}$.

En efecto, al utilizar la metodología de cálculo para el factor de restricción de la EFC basada en la Ec. (6), los valores de $\mathrm{P} / \sigma_{\mathrm{o}}$, evaluados a $\mathrm{v}_{\mathrm{N}}=0,002$ y divididos por la función $\mathrm{G}$ y el respectivo valor del límite elástico, darán los valores buscados de $\Omega^{*}$. Al repetir esta estrategia con todos los valores de a/W y espesor $B$, se obtiene las curvas de $\Omega^{*}$ en función del espesor equivalente, $\mathrm{B} / \mathrm{b}$, tanto para las probetas MB como para las MS, mostradas en la figura 7.

Los datos mostrados en la figura 7 resultan interesantes. En primer lugar, las dos curvas no son coincidentes, salvo en el sector en que $\mathrm{B} / \mathrm{b}$ tiende al valor 2,5 . Este punto equivale a a/W $=0,8$, y $B$ $=25 \mathrm{~mm}$. Como consecuencia, en ambas probetas, MB y MS, el grado de constricción es elevado; ambos valores son muy parecidos, y cercanos (por exceso) al límite teórico superior de 0,364 (deformación plana). Luego, para probetas con grieta profunda y espesor grande, el factor de constricción, $\Omega^{*}$, no parece ser sensible a la presencia de metal de soldadura.

Un segundo elemento de interés de la figura 7, es que, en la medida que $\mathrm{B} / \mathrm{b}$ disminuye (esto es, el tamaño de grieta y/o el espesor disminuyen), también disminuye el valor de $\Omega^{*}$ para ambos tipos de probeta. Las probetas de MS, sin embargo, sufren

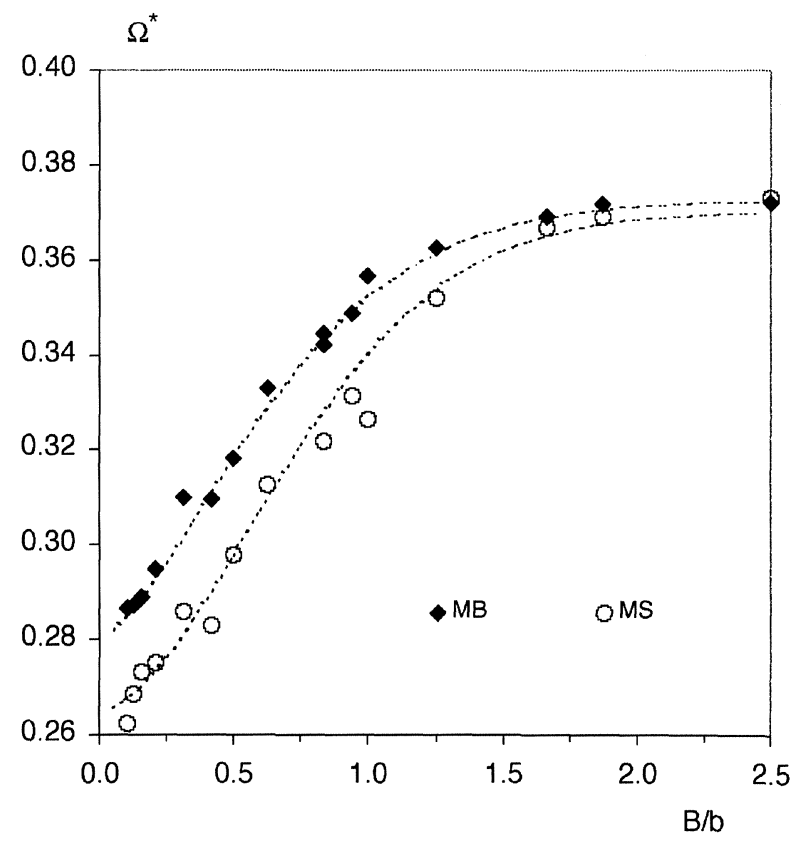

Figura 7. El comportamiento de $\Omega^{*}$ para probetas de $M B$ $y$ de $M S$, en función de $B / b$.

Figure 7. The behavior of $\Omega^{*}$ for $M B$ and $M S$ specimens, as a function of $B / b$. 
una disminución en $\Omega^{*}$, más rápida que las de $\mathrm{MB}$, en la medida que disminuye el valor de $\mathrm{B} / \mathrm{b}$. Por ello, el factor de constricción de MS llega a un valor mínimo de 0,262, a B/b =0,104. Este valor de $\Omega *$ es parecido al límite inferior (tensión plana), en tanto el factor de constricción para $\mathrm{MB}$ es algo mayor, con un valor de 0,285 , a igual espesor equivalente.

De lo anterior se puede inferir la consecuencia fundamental que conlleva la presencia de metal de soldadura en la probeta bimetálica $\mathrm{C}(\mathrm{T})$ : provoca una disminución del grado de constricción respecto de una probeta con, sólo, metal base. Este efecto es tanto mayor cuanto menor es la longitud de grieta y/o el espesor de la probeta.

Donoso et al. ${ }^{[14]}$ han señalado que el factor de constricción de la EFC está vinculado a la fracción de material bajo condiciones de deformación plana. Luego, un valor elevado de $\Omega^{*}$, cercano al límite superior de 0,364 , indicará una condición que tiende a deformación plana. A la inversa, un valor bajo de $\Omega^{*}$, cercano a 0,268 , corresponderá a una situación que tiende a tensión plana. Como se ha sugerido $^{[14]}$, siempre habrá una fracción de la probeta, aún cuando sea pequeña, que estará bajo condiciones de tensión plana (las caras laterales, externas, de la probeta plana). De otra parte, una condición pura de deformación plana no podrá existir, al menos no para el $100 \%$ de la probeta, puesto que tensión plana siempre estará presente.

En este contexto, disminuir la constricción en la probeta $\mathrm{C}(\mathrm{T})$ implica disminuir la cantidad de material bajo una condición de deformación plana. Para la probeta $\mathrm{MB}$, se llega a esta condición mediante un cambio de las dimensiones relevantes de la probeta, de manera que $\mathrm{B} / \mathrm{b}$ disminuya. Este efecto se logra, ya sea disminuyendo el espesor o aumentando el tamaño del ligamento, lo cual es equivalente a disminuir el tamaño de grieta.

En la probeta MS, sin embargo, la disminución en el grado de constricción, medido por $\Omega^{*}$, provocada originalmente por la disminución en el espesor efectivo, $\mathrm{B} / \mathrm{b}$, se ve intensificada por el efecto de la constricción por el material. En efecto, para el mismo valor de $\mathrm{B} / \mathrm{b}$, el factor de constricción de las probetas MS disminuye más allá del valor determinado sólo por el espesor equivalente. Una interpretación de este hecho, a la luz del significado de $\Omega^{*}$ antes comentado, es que la presencia del metal de soldadura sobre-ajustado, rodeando la grieta, contribuye a disminuir la fracción efectiva de material en un estado de deformación plana, cuando se le compara con probetas de sólo metal base. De hecho, probetas de MS, con $\mathrm{B} / \mathrm{b} \leq 0,5$ tienen un valor del factor de constricción que indica un estado de tensión plana dominante.

El significado de esta condición puede descifrarse analizando la distribución de la tensión de abertura en la punta de la grieta, análisis que puede hacerse, sólo, numéricamente. La presencia de MS altera la distribución de tensiones alrededor de la punta de la grieta en la probeta respecto de una referencia, que puede ser una probeta con, sólo, MB. El análisis corresponde al llamado enfoque de dos parámetros o modelo J-Q de O'Dowd y Shih $^{[18}$ y 19] . En su tesis ${ }^{[20]}$, Ortiz aplicó el modelo $\mathrm{J}-\mathrm{Q}$ a probetas $\mathrm{MB}$ y MS, evaluando las tensiones de abertura en el ligamento para algunos de los casos presentados en este trabajo. El efecto de la presencia de MS se muestra en la figura 8 , mediante las curvas de tensión de abertura normalizada $\sigma_{\mathrm{yy}} / \sigma_{\mathrm{o}}$ para MB y MS en el centro de la probeta (correspondiente a $z=0$; ver Fig. 2), en función de la coordenada de distancia normalizada $\mathrm{x} /\left(\mathrm{J} / \sigma_{\mathrm{o}}\right)$.

En la figura 8, las curvas correspondientes a $\mathrm{MB}$ y $\mathrm{MS}$, para $\mathrm{B} / \mathrm{b}=0,156$ y $\mathrm{B} / \mathrm{b}=1,667$, se comparan con las respectivas curvas para la tensión HRR ${ }^{[21}$ y 22$]$. Las curvas $\sigma_{\text {yy }} / \sigma_{\mathrm{o}}$ de MS se apartan más de la curva HRR que las de $M B$, en la medida que el espesor equivalente $\mathrm{B} / \mathrm{b}$ disminuye. La diferencia entre la tensión de abertura normalizada, $\sigma_{\mathrm{yy}} / \sigma_{\mathrm{o}}$ y la tensión HRR normalizada se conoce

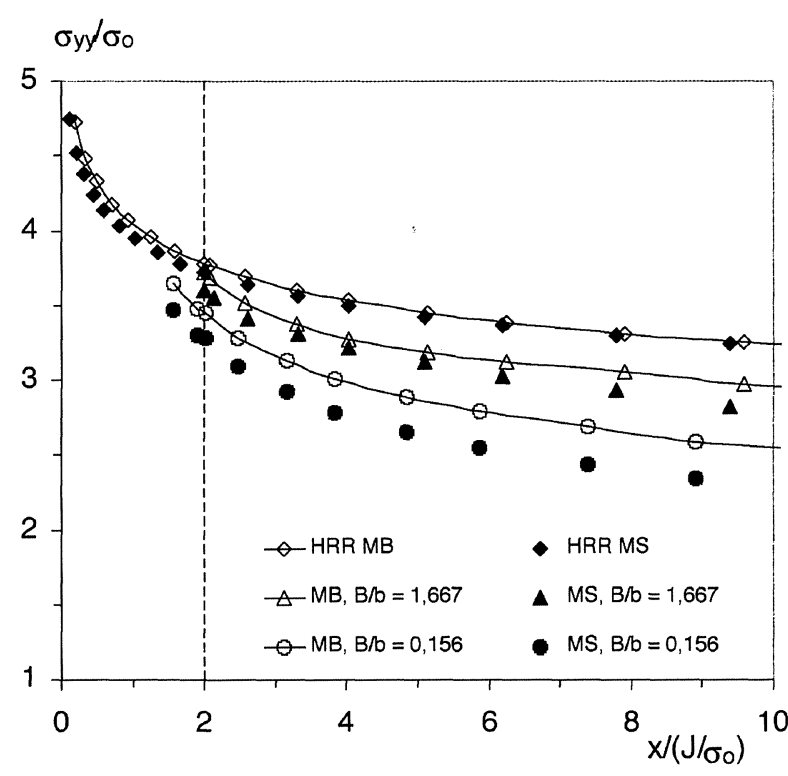

Figura 8. Tensión de abertura normalizada, $\sigma_{y y} / \sigma_{0}$ y tensión $H R R$ de $M B$ y MS, para dos valores de espesor equivalente, $B / b^{[22]}$.

Figure 8. Experimental data and tendency curves for $M B$ and MS. 
como parámetro $Q$ y es una medida del grado de constricción del componente agrietado. En el contexto del enfoque $\mathrm{J}-\mathrm{Q}^{[18}$ y $^{19]}$, esto implica que la constricción en la probeta bi-metálica disminuye más que en aquella que tiene sólo $\mathrm{MB}$, en función del espesor equivalente $B / b$. En este sentido, $Q$ y $\Omega^{*}$ describen el mismo fenómeno, de diferente manera ${ }^{[20]}$.

Los enfoques citados al inicio, J-Q-M ${ }^{[7]}$, modificación del modelo J-Q o ETM ${ }^{[8 \text { y 9] }}$, son de carácter estrictamente bidimensional y han mostrado que la presencia del metal de soldadura provoca un cambio en el grado de constricción respecto a una referencia, la cual puede ser tomada arbitrariamente como, sólo, metal base o, sólo, metal de soldadura. En el enfoque J-Q-M suele escogerse como referencia a la geometría constituida de material de soldadura homogéneo cuando la grieta se ubica en el cordón de soldadura ${ }^{[23}$ y 24]. La razón de esto es que, a cargas bajas, el tamaño de la zona plástica es pequeño respecto al ancho del cordón, por lo que el campo de tensiones delante de la punta de la grieta actúa como si se tratara únicamente de material de soldadura homogéneo, no apreciándose los efectos del desajuste de material $(\mathrm{M}=0)$. Cuando el tamaño de la zona plástica alcanza el orden de magnitud del tamaño del cordón, comienza a aparecer el efecto del desajuste de material. En el caso específico de sobre-ajuste, $\mathrm{M}$ se hace más negativo a medida que aumenta la carga, dando así lugar a una pérdida de constricción.

El grado de constricción en el ETM ${ }^{[8 \text { y } 9]}$ se refleja en el aumento o disminución de la carga límite del sistema bi-material respecto de un sistema constituido por metal base homogéneo. Para el caso de sobre-ajuste, la carga límite del sistema bimaterial es mayor que la del sistema constituido de metal base homogéneo, pero menor que la de un sistema constituido de sólo metal de soldadura homogéneo. En la medida que aumenta el grado de sobre-ajuste $(\mathrm{MM}>1)$, estas diferencias se acentúan.

No es fácil comparar con otros trabajos sobre constricción causado por el material, dado que el análisis de la constricción fuera del plano con el factor $\Omega^{*}$ ha sido privativo de este grupo de investigación. A modo de ejemplo, el artículo de Donoso, Cancino y Muehlich ${ }^{[14]}$ mostró el comportamiento del factor $\Omega^{*}$ en probetas $\mathrm{DC}(\mathrm{T})$ para un material homogéneo (equivalente a metal base, MB). De otra parte, el trabajo de Donoso y Landes a partir del cual se generó el factor $\Omega^{*}{ }^{[2]}$, contiene información sobre tres aceros (también equivalen- tes a metal base), del cual se obtuvo el comportamiento de $\Omega^{*}$ en función de $\mathrm{B} / \mathrm{b}$.

Antecedentes limitados del comportamiento del metal de soldadura pueden encontrarse en el trabajo de Donoso, Labbé y Mora ${ }^{[25]}$, a partir de los cuales se ha confeccionado el gráfico de la figura 9 . En ella, se muestran datos experimentales ${ }^{[2]}$ incluidos en otros textos ${ }^{[14]}$, marcados como "D\&L" (equivalentes a MB), junto con valores de $\Omega *$ para un acero ASTM A588 (MB) y su unión soldada (MS) con un sobre-ajuste $M M=1,48^{[25]}$. Los datos D\&L y A588, MB y MS, permiten confirmar el comportamiento idealizado mostrado en la figura 7 , aún cuando correspondan a un sobre-ajuste diferente, muestren algunas desviaciones y no cubran todo el rango de valores del espesor equivalente $\mathrm{B} / \mathrm{b}$.

Una comparación interesante entre los dos tipos de probeta, MB y MS, es el del comportamiento a igual $\Omega^{*}$. Para ello, se ha seleccionado el caso $\Omega^{*} \approx 0,307$, que comprende a la probeta $\mathrm{MB}$ con $\mathrm{B} / \mathrm{b}=0,417(\mathrm{a} / \mathrm{W}=0,4$ y $\mathrm{B}=12,5 \mathrm{~mm})$, y a la probeta MS con $\mathrm{B} / \mathrm{b}=0,625(\mathrm{a} / \mathrm{W}=0,8$ y $\mathrm{B}=$ $6,25 \mathrm{~mm}$ ). La figura 10 muestra curvas carga normalizada vs. desplazamiento normalizado para ambas probetas, en las cuales $\mathrm{P}_{\mathrm{N}}$ ha sido dividido además por el correspondiente límite elástico. Para efectos de comparación, la curva de la probeta MS con igual espesor equivalente que la probeta de

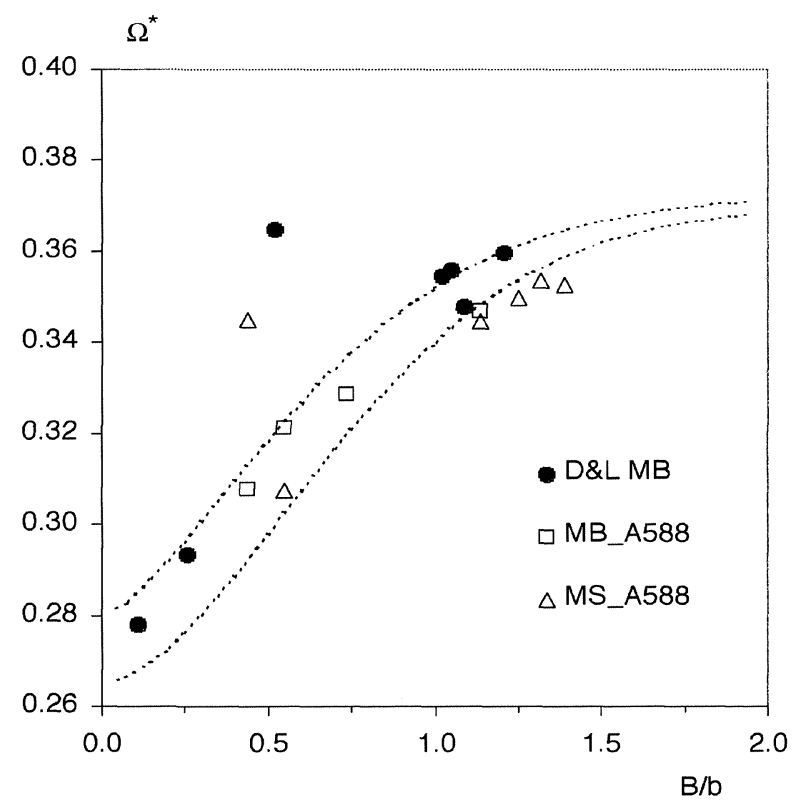

Figura 9. Datos experimentales y curvas de tendencia para $M B$ y $M S$.

Figure 9. Experimental data and tendency curves for $M B$ and MS.

Rev. Metal. Madrid 39 (2003) 357-366 


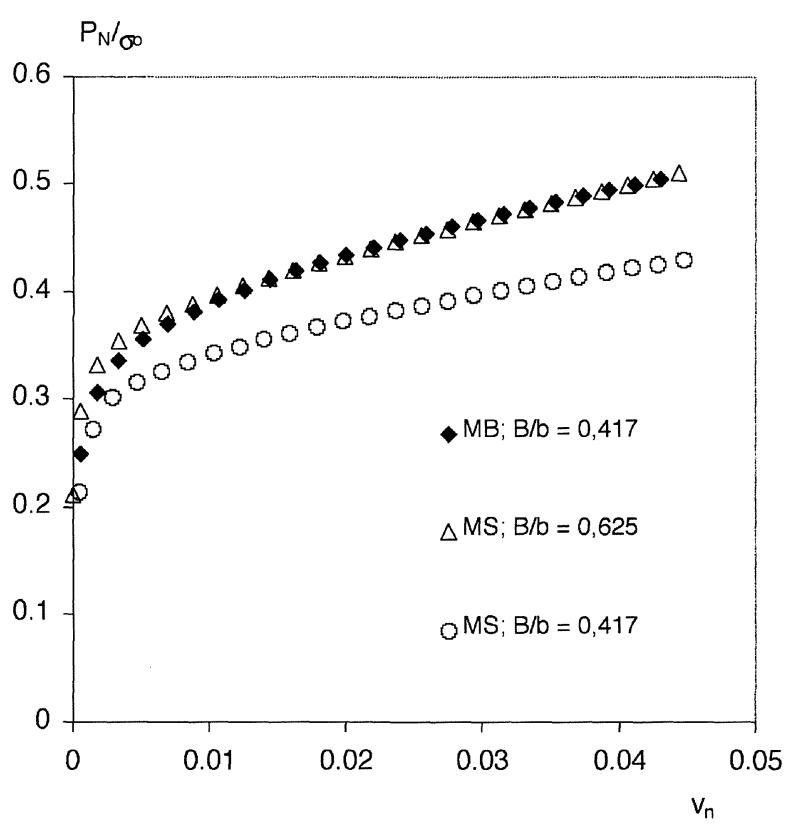

Figura 10. Comparación del comportamiento a igual $\Omega^{*} \mathrm{e}$ igual $B / b$.

Figure 10. Comparison of the behavior at the same values of $\Omega^{*}$ and $B / b$.

$\mathrm{MB}(\mathrm{B} / \mathrm{b}=0,417)$ ha sido incluida en esta figura ( $\mathrm{a} / \mathrm{W}=0,7 ; \mathrm{B}=6,25 \mathrm{~mm}, \mathrm{y} \Omega^{*} \approx 0,282$ ). Como se puede observar en la figura 10 , las curvas de $\mathrm{MB}$ y MS para igual factor de constricción $\left(\Omega^{*} \approx 0,307\right)$ son prácticamente coincidentes. Al comparar las curvas para espesor equivalente de 0,417, la de MS, que tiene un factor de constricción menor, 0,282, está ubicada por debajo de las otras dos curvas.

Una de las características más relevantes del factor de constricción de la EFC, es que permite obtener curvas verdaderamente normalizadas. En efecto, al dividir carga normalizada, $\mathrm{P}_{\mathrm{N}}$, por $\Omega^{*}$, de la ecuación (3), se obtiene,

$$
\mathrm{P}_{\mathrm{N}}=\sigma^{*} \cdot\left(\mathrm{v}_{\mathrm{N}}\right)^{1 / \mathrm{n}}
$$

donde, $\mathrm{P}{ }_{\mathrm{N}}$ es la carga verdaderamente normalizada. La ecuación (8) indica que $\mathrm{P}^{*} \mathrm{~N}$, como función del desplazamiento normalizado, depende sólo de las propiedades de deformación plástica del material, $\mathrm{n}$ y $\sigma^{*}$. En este contexto, la curva $\mathrm{P}_{\mathrm{N}}{ }^{-} \mathrm{v}_{\mathrm{N}}$ es propia del material. Más aún, la carga verdaderamente normalizada al inicio de deformación plástica $\left(v_{N} \approx 0,002\right), P^{*}{ }_{N o}$, resulta ser igual al límite elástico del material ${ }^{[14]}$.

La figura 11 muestra el proceso de normalización verdadero para las mismas probetas analizadas en las figuras 4 y $5(\mathrm{MB})$, a las que se han agregado

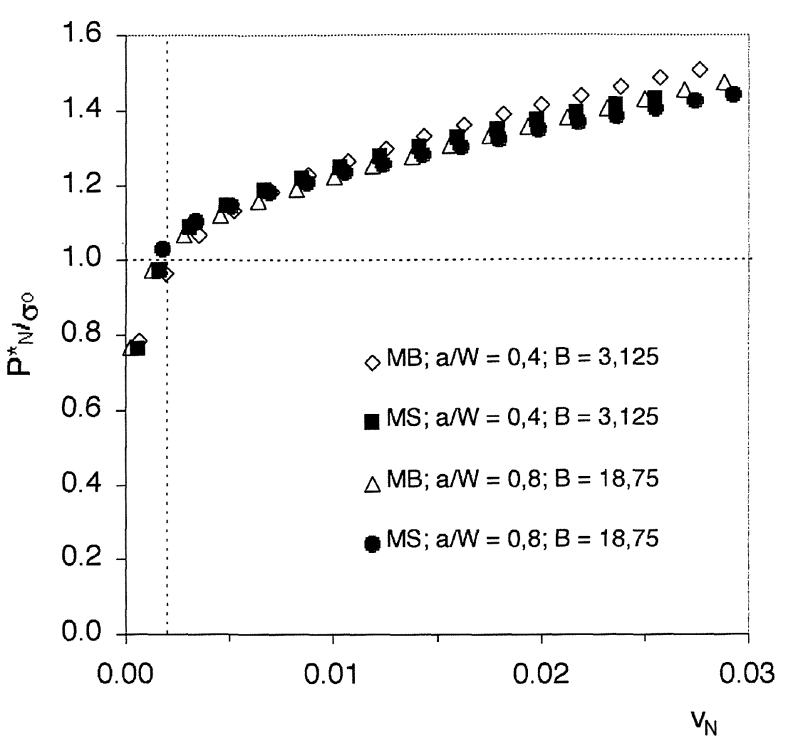

Figura 11. Curvas verdaderamente normalizadas.

Figure 11. Truly normalized curves.

sus análogas para MS para ilustrar este efecto. La línea segmentada vertical de la figura 11 ha sido trazada a $v_{N}=0,002$, para señalar así que la intersección con cada curva, al valor de la ordenada igual a 1, línea segmentada horizontal, se produce al valor del respectivo límite elástico.

\section{CONCLUSIONES}

- El factor de constricción $\Omega^{*}$ de la Ecuación del Formato Común ha sido usado para evaluar numéricamente el efecto de la constricción por el material en probetas de fractura $C(T)$, con valores del espesor B entre 3,125 y $25 \mathrm{~mm}$ y longitudes de grieta normalizadas, a/W, entre 0,4 y 0,8 .

- El factor de constricción $\Omega^{*}$ depende del espesor equivalente $\mathrm{B} / \mathrm{b}$, disminuyendo en la medida que $\mathrm{B} / \mathrm{b}$ decrece. La disminución de $\mathrm{B} / \mathrm{b}$ se logra al disminuir B o al aumentar el ligamento (esto es, para grietas pequeñas).

- El factor de constricción $\Omega^{*}$ tiende al valor propio de deformación plana para valores grandes de B/b. En el caso presentado aquí, esta situación se dará, probablemente, para $\mathrm{B} / \mathrm{b} \geq 1$ y parece no estar afectado por la presencia de MS.

- A la inversa, $\Omega^{*}$ tiende al valor de tensión plana $(0,268)$ en la medida que $B / b$ decrece. Esta tendencia, sin embargo, parece depender fuertemente de la presencia de metal de soldadura. Así, las probetas de MS muestran una 
disminución más rápida en $\Omega^{*}$ que las probetas de MB.

- Como consecuencia de esto, la presencia de MS en la probeta $C(T)$, en una fracción en volumen de 0,20, y con un grado de sobreajuste del límite elástico de 1,25, logra disminuir la constricción al comparar con la probeta $\mathrm{C}(\mathrm{T})$ con sólo MB. Este efecto de constricción por el material es mayor en la medida que $\mathrm{B} / \mathrm{b}$, el espesor equivalente, disminuye.

\section{Agradecimientos}

Este trabajo está basado parcialmente en la Tesis de Ingeniero Civil Mecánico del Sr. Alejandro Ortiz Bernardin. Los autores agradecen el financiamiento del Proyecto de Investigación FONDECYT-Chile 1010151, y del Proyecto U.T.F.S.M-DGIP 210121.

\section{REFERENCIAS}

[1] W. BRocks y W. SCHMITT, ASTM STP 1244, Mark Kirk and Ad Bakker (Eds.), American Society For Testing and Materials, Philadelphia, 1995, pp. 209-231.

[2] J.R. DONOSO y J.D. LANDES, Int. J. Fract. 63 (1993) 275 285.

[3] N.P. O'Dowd y C.F. SHIH, ASTM STP 1207, John D. Landes, Donald E. McCabe and J.A.M. Boulet (Eds.), American Society For Testing And Materials, Philadelphia, 1994, pp. 21-47.

[4] J.R. Donoso, M. Bindhoff y F. LABbÉ, 2001 Summer Computer Simulation Conference, Orlando, 2001.

[5] K.H. Schwalbe y M. KoçAK (Eds.), Mis-Matching of Welds, ESIS Publication 17, 1994.

[6] K.H. Schwalbe y M. KoçAK (Eds.), Mis-Matching of Interfaces and Welds, GKSS Research Center Publications, 1997.

[7] C. Thaulow, Z.L. Zhang, $\varnothing$. Ranestad y M. Hauge, Fatigue and Fracture Mechanics: $30^{\text {th }}$ Volume, ASTM STP 1360, P.C. Paris and K.L. Jerina (Eds.), American Society
For Testing and Materials, West Conshohocken, PA, 2000, pp. 102-114.

[8] Y.J. Kim y K.H. Schwalbe, Eng. Fract. Mech. 68 (2001) 1.137-1.151.

[9] Y.J. Kim y K.H. Schwalbe, Eng. Fract. Mech. 68 (2001) 163-182.

[10] MoltubakK, C. Thaulow, y Z.L. Zhang, Eng. Fract. Mech. 62 (1999) 445-462.

[11] J.R. Donoso y J.D. Landes, Eng. Fract. Mech. 47-5 (1994) 619-628.

[12] J.R. Donoso y J.D. Landes, Eng. Fract. Mech. 54-4 (1996) 499-512.

[13] J.R. Donoso y J.D. LANDES, in Fatigue and Fracture Mechanics: $32^{\text {nd }}$ Volume, ASTM STP 1406, R. Chona (Ed.), American Society For Testing and Materials, West Conshohocken, PA, 2000, pp. 261-278.

[14] J.R. Donoso, C. Cancino y U. Muehlich, Rev. Metal. Madrid 38 (2002) 326-333.

[15] J.R. Donoso, U. Muehlich y J.D. LANDES, Fracture Mechanics Beyond 2000, Vol. I, Krakow, Poland, 2002, pp. 497-504.

[16] ADINA, Theory and Modeling Guide, Vol. 1, ADINA, ADINA R\&D, Inc., 2001.

[17] Standard Test Method for Measurement of Fracture Toughness, ASTM E1820-99a, ASTM Annual Book of Standards, Vol. 03-01.

[18] N.P. O’Dowd y C.F. SHiH, J. Mech. Phys. Solids 39 (1991) 898-1.015.

[19] N.P. O'Dowd y C.F. SHiH, J. Mech. Phys. Solids 40 (1992) 939-963.

[20] A. Ortiz, Tesis de Ingeniero Civil Mecánico, Universidad Técnica Federico Santa María, Octubre 2002.

[21] J.R. Rice y G.F. Rosengren, J. Mech. Phys. Solids 16 (1968)1-12.

[22] J.W. Hutchinson, J. Mech. Phys. Solids 16 (1968) 13-31.

[23] M.C. Burstow, I.C. Howard y R.A. Ainsworth, J. Mech. Phys. Solids 5 (1998) 845-872.

[24] M.C. Burstow, I.C. Howard y R.A. Ainsworth, Int. J. Fract. 89 (1998) 117-142.

[25] J.R. Donoso, F. Labbé y C. Mora, Eng. Fract. Mech. 4 (1993) 515-520. 